\title{
Vaginal reconstruction with the Abbe-McIndoe technique in Mayer Rokitansky Kuster Hauser syndrome: a case report
}

\section{Sujata Swain ${ }^{1}$, Abarajda Dilip ${ }^{1}$, Prithviraj Singh ${ }^{2}$, Rabi Narayan Satpathy ${ }^{1}$}

\author{
${ }^{1}$ Department of Obstetrics \& Gynaecology, S.C.B. Medical College, Cuttack, Odisha, India \\ ${ }^{2}$ Department Plastic Surgery, S.C.B. Medical College, Cuttack, Odisha, India
}

Received: 23 July 2015

Accepted: 14 August 2015

\author{
*Correspondence: \\ Dr. Sujata Swain, \\ E-mail: sjt_swain@yahoo.co.in
}

Copyright: $(\mathcal{C}$ the author(s), publisher and licensee Medip Academy. This is an open-access article distributed under the terms of the Creative Commons Attribution Non-Commercial License, which permits unrestricted non-commercial use, distribution, and reproduction in any medium, provided the original work is properly cited.

\begin{abstract}
The Mayer-Rokitansky-Küster-Hauser (MRKH) syndrome is a rare but well discussed entity characterized by congenital aplasia of the uterus and the upper part $(2 / 3 \mathrm{rd})$ of the vagina in women with normal development of secondary sexual characteristics and a normal 46, XX karyotype. The inability to lead a normal sexual and reproductive life bears a great amount of social inhibition and psychological distress on the affected individuals. The physical inability can definitely be attended to by various non-surgical and surgical techniques leading to a free sexual life. We report a case of MRKH who underwent an Abbe-McIndoe technique with one year follow up. The patient due to social inhibition, failed to report primary amenorrhea at an earlier age. She seeked medical help at the time of marriage at the age of $30 \mathrm{yrs}$.Successful vaginal reconstruction with minimal expenditure and counselling paved the way for her marriage and satisfactory sexual life.
\end{abstract}

Keywords: Mayer-Rokitansky-Küster-Hauser syndrome, Aplasia of the uterus, Primary amenorrhea, Abbe-McIndoe procedure, Neovagina, Split skin autograft

\section{INTRODUCTION}

Mayer-Rokitansky-Kuster-Hauser (MRKH) syndrome consists of vaginal aplasia with other mullerian (i.e., paramesonephric) duct abnormalities. ${ }^{1}$ Type I MRKH is less common than Type II MRKH, also known as MURCS (Mullerian, Renal Cervical Somite). The latter is associated with Renal (unilateral agenesis, ectopia of kidneys or horseshoe kidney) skeletal and vertebral (Klippel-Feil anomaly; fused vertebrae, scoliosis) or hearing defects. ${ }^{2}$ Type I Mayer-Rokitansky-KusterHauser syndrome is characterized by an isolated absence of the proximal two thirds of the vagina. The MRKH syndrome is also referred to as CAUV (Congenital Absence of the Uterus and Vagina), MA (Müllerian Aplasia) or GRES (Genital Renal Ear Syndrome). Following diagnosis, intervention allows patients to have normal sexual function. Reproduction may be possible with assisted techniques depending on the extent of agenesis.

\section{CASE REPORT}

A 30 year old unmarried lady presented to us with primary amenorrhea. Due to social inhibitions, this was the first medical consultation she had ever made .Her basic worries were whether she could lead a normal sexual and reproductive life if she gets married. On clinical examination she was found to have well developed secondary sexual characteristics and a vaginal dimple. Ultrasonography and magnetic resonance imaging (MRI) revealed the absence of a uterus and normal ovaries were present. An ultrasound KUB did not reveal any renal abnormalities. Screening for skeletal and otological anomalies were done and found to be negative. She was diagnosed with type 1 MRKH. The clinical scenario was explained to the patient who was anxiously awaiting marriage. She underwent proper counselling, and thereafter surgical options were discussed. Since there was complete vaginal agenesis 
non-surgical option was not applicable. She had normal circulating levels of luteinizing hormone (LH) and follicle-stimulating hormone (FSH) indicating normal ovarian function. Having ruled out other endocrine abnormalities, after a thorough preoperative evaluation she was posted for Abbe-Mcindoe procedure. The plan was to create neovagina lined by split skin autograft.

The procedure consisted of three steps. First was to create safely a new cavity between the rectum and the bladder in the correct anatomical plane. Second step was to prepare an airtight mould over which dermal graft was to be secured. Third step was to insert and secure the graftmould assembly into the neovagina. The procedure was done under spinal anaesthesia. The patient was placed in lithotomy position and catheterised (Figure 1). The catheter helps in defining urethral margins clearly during the procedure. Neovagina was created safely by dissecting initially on either side of the midline and creating a space between the bladder and rectum by sequentially inserting dilators of grater diameters for a depth of around $6 \mathrm{~cm}$.After adequately creating space, the median remnant raphe was excised. Haemostasis was achieved and a mop soaked in adrenaline solution was placed in the cavity (Figure 2).

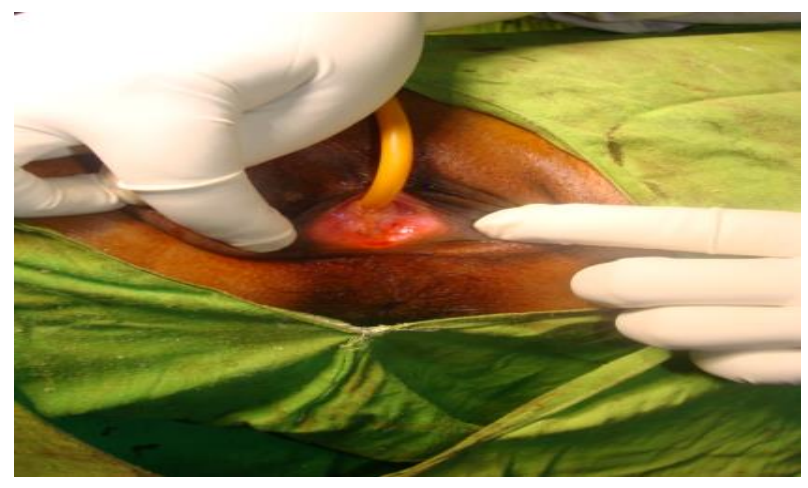

Figure 1: Pre-operative vaginal dimple.

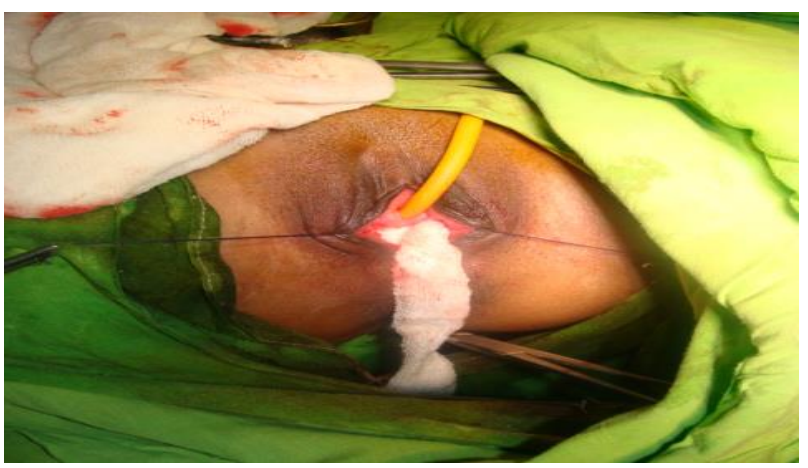

Figure 2: After creating new cavity.

A sterile sponge was rolled around a PVC nelcath catheters conical end which has two lateral eyes and the ends of the sponge were sutured with no 2 silk (Figure 2). A thick condom was placed over this mould and tied at one end so that the mould is now airtight to make it collapsible (Figure 3). Thiersch skin autograft was harvested from medial aspect of thigh (Figure 4). Multiple splits were made and washed thoroughly in saline mixed with antibiotic solution (Figure 5). Paraffin gauze was applied over the condom so that mould could be removed easily later (Ovulatory dysfunction is one of the most common causes of reproductive failure in subfertile and infertile couples. The most common type of ovulatory dysfunction is anovulation. Polycystic ovarian syndrome (PCOS) is thought to be the commonest cause of anovulatory infertility.

Various medications are used for induction of ovulation among infertile patients with PCOS. The first line oral treatment is non-steroidal selective estrogen receptor modulators (SERM). The structural similarity to estrogen allows SERMs to bind to estrogen receptors(ER) throughout the reproductive system; however, in contrast to estrogen, SERMs binds nuclear ER for an extended period of time, which consequently depletes ER concentrations by interfering with the normal process of ER replenishment. Clomiphene citrate and tamoxifen are commonly used SERMs to induce ovulation. ${ }^{2}$

In the clinical setting of anovulation, SERM are thought to act primarily by binding with estrogen receptors at the hypothalamus. The competitive inhibition results in a perceived drop in endogenous estrogen levels. Depletion of hypothalamic ER prevents correct interpretation of circulating estrogen levels which is perceived falsely as low. Such misinterpreted reduced levels of estrogen negative feedback lead to normal compensatory mechanisms. This alters the pattern of pulsatile hypothalamic gonadotropin-releasing hormone (GnRH) secretion to stimulate increased secretion of pituitary gonadotropins that, in turn, serve to drive ovarian follicular activity. In anovulatory women with PCOS, in whom the GnRH pulse frequency is already abnormally high, SERMs treatment was found to increases pulse amplitude, but not frequency.

Since its introduction in 1956, clomiphene citrate has been the first-line method of ovulation induction in couples with anovulatory infertility. Many studies have shown that approximately $80 \%$ of women ovulate while using clomiphene citrate but only $40 \%$ of women achieve pregnancy. Many authors have proposed that this discrepancy in ovulation and pregnancy rate is due to the antiestrogenic effects of clomiphene, especially on the uterus, cervix and vagina, resulting in a thin endometrial lining and poor cervical mucus. Apart from serious side effects like ovarian hyperstimulation syndrome, studies have reported concerns that use of clomiphene citrate is associated with increased chances of ovarian cancer. ${ }^{5,6}$

Recently another SERM, tamoxifen, has also been used to induce ovulation. It is primarily used as an adjuvant therapy in the treatment of breast cancer but its use as an ovulatory agent was first reported in $1973 .^{7}$ 
Unlike clomiphene, tamoxifen acts as an agonist on the estrogen receptors of the vaginal mucosa and endometrium but studies on the effects of tamoxifen on cervical mucus have been inconclusive. Also there are no reports of increased chances of ovarian cancer with use of tamoxifen. Even though its use has been shown to increase risk for endometrial cancer but with short term use that possibility is unlikely. Various randomized controlled trials found that tamoxifen is as effective as clomiphene citrate in inducing ovulation. Despite a trend toward improved pregnancy rates with tamoxifen, further studies are necessary to confirm its efficiency and safety. ${ }^{8}$

This prospective randomized trial was planned to find out the safety and efficacy of tamoxifen in patients of PCOS with primary or secondary anovulatory infertility (Figure 6 ). With the graft spread over the mould with raw surface facing outward, the ends of the graft were sutured (Figure 7 and 8). Now the mop was removed from the cavity and haemostasis reassured. The graft mould assembly was inserted into the cavity and labia majora were sutured to keep the mould in position (Figure 9 and 10).

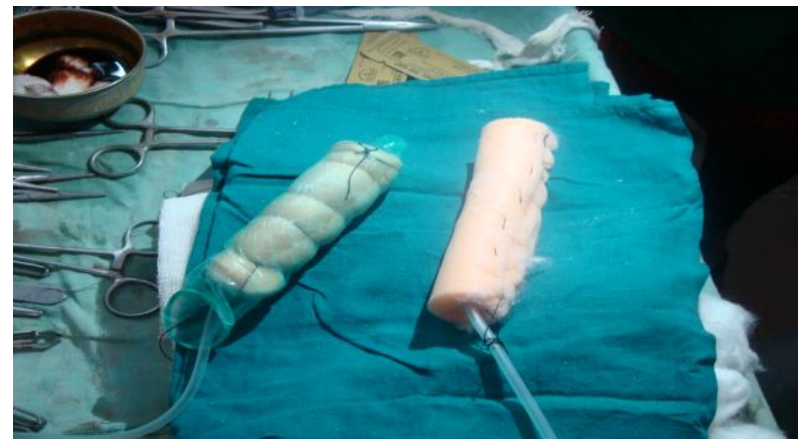

Figure 3: Sponge sutured around catheter and placed in a condom.

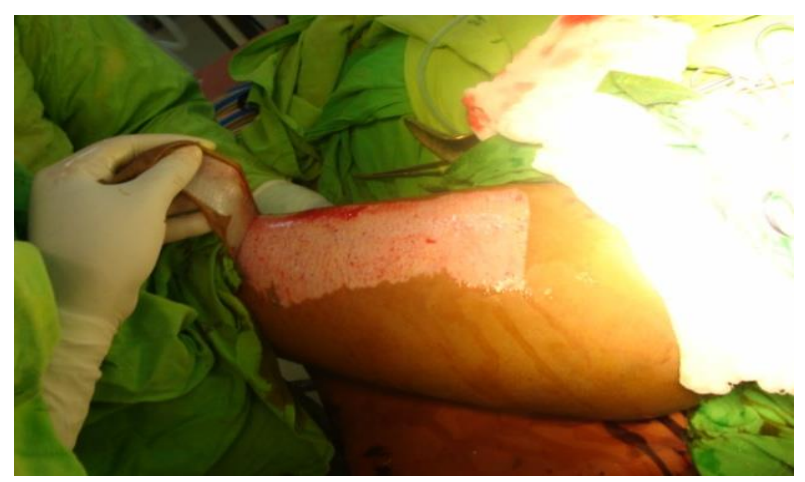

Figure 4: Harvesting graft.

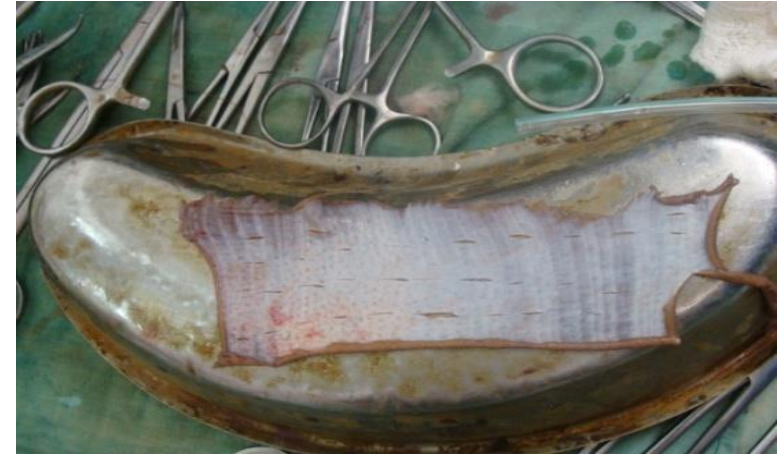

Figure 5: Prepared split dermal autograft.

Postoperative period was uneventful and managed with adequate hydration, analgesics and broad spectrum antibiotics for three days. Liquid diet was started six hours following surgery. Semisolid diet was recommended for three days. After a week, the labial sutures were removed and the mould was removed carefully. Thorough saline wash was done and the graft was inspected for take up. Graft took up well and we changed the moulds every third day until three weeks. She was asked to use rubber moulds regularly and we followed her up every six weeks. We explained her fiance the medical, sexual and reproductive possibilities and. At her one year follow up six months since marriage, the happy couple reported with satisfactory sexual life and have planned surrogacy.

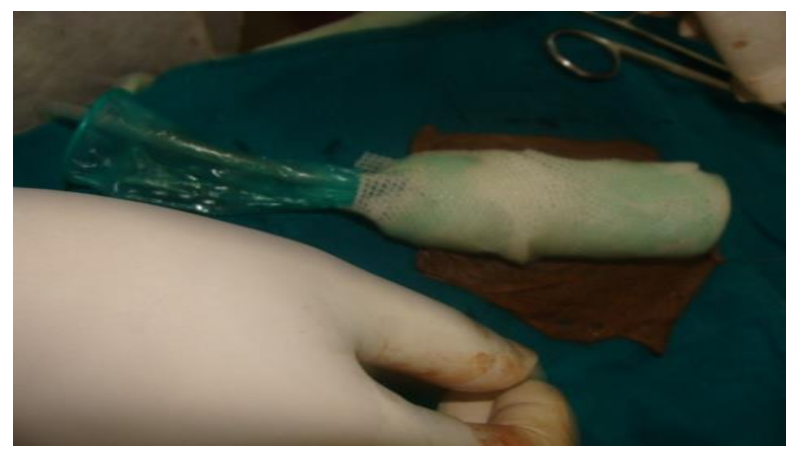

Figure 6: Paraffin gauge around the mould.

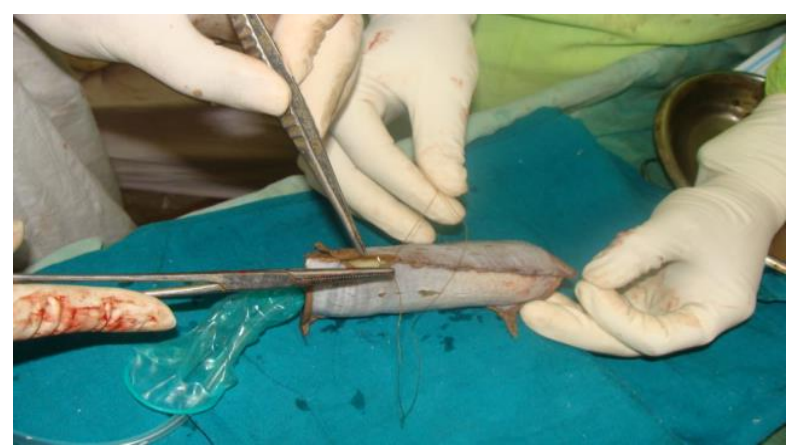

Figure 7: Graft being secured over the mould. 


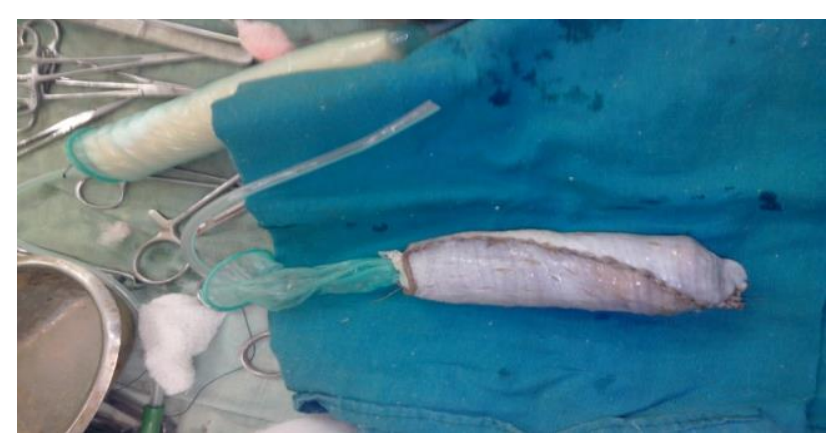

Figure 8: Graft-mould assembly.

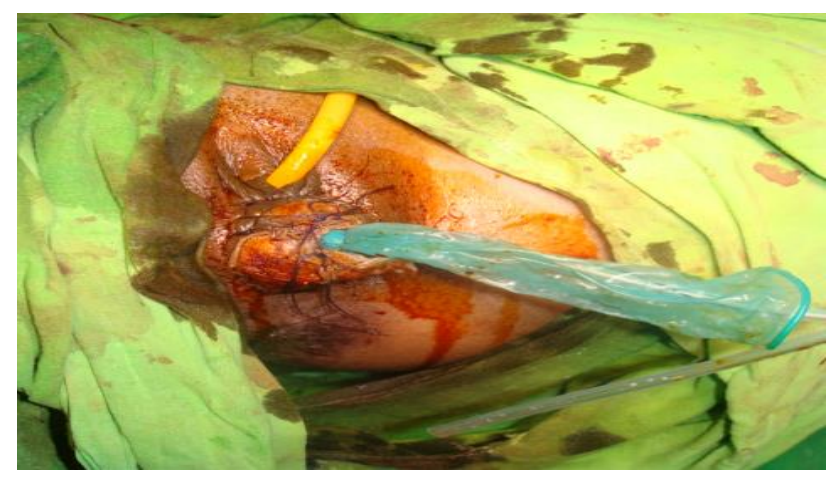

Figure 9: Labia majora sutured.

\section{DISCUSSION}

Hauser and Schreiner in 1961 first described the MRKH syndrome after reviewing the autopsy reports described by Mayer in 1829, Rokitansky in 1838 and Kuster in $1910 .^{3}$ MRKH syndrome is a rare but well discussed entity with incidence of 1 in 4500 female births characterized by congenital aplasia of the uterus and the upper part $(2 / 3)$ of the vagina in women with normal development of secondary sexual characteristics. First clinical signal is generally a primary amenorrhea in patients presenting with a normal female phenotype, normal 46, XX karyotype, ${ }^{4-7}$ and normal functioning ovaries with no sign of androgen excess. ${ }^{8,9}$ Physical examination reveals completed puberty with normal secondary female sexual characteristics (pubic hair and breast development are Tanner stage ) and normal external genitalia. At the same time, the vagina is reduced to a more or less deep $(2-7 \mathrm{~cm})$ vaginal dimple.

Anatomic examination is however necessary to diagnose an MRKH syndrome of either type. Complete uterus aplasia in the presence of two rudimentary horns linked by a peritoneal fold and normal Fallopian tubes is isolated or MRKH type I syndrome. ${ }^{10}$ Type II MRKH is characterized by uterine symmetry or asymmetric hypoplasia, accompanied by aplasia of one of the two horns or by a size difference between the two horn rudiments, coupled with tubal malformations such as hypoplasia or aplasia of one or the two tubes. ${ }^{11}$

Transabdominal ultrasound, magnetic resonance imaging, skeletal survey, phenotyping and endocrinological profiling are usually done for achieving or conforming diagnosis. Celioscopy is an invasive procedure done in patients with doubtful diagnosis who are willing for therapeutic intervention.

The only nonsurgical option is frank dilatation technique successful used in motivated patients willing to spend time to create a neovagina using the moulds. Abbe Mcindoe technique and its various modifications, Williams vaginoplasty, rotational flap procedures, intestinal neovagina, Vecchietti technique are the various surgical techniques described so far. ${ }^{12-14}$

\section{CONCLUSION}

The inability to lead a normal sexual and reproductive life bears a great amount of social inhibition and psychological distress on the affected individuals. The physical inability can definitely be attended to by nonsurgical and various surgical techniques leading to a distress free sexual life. The surgical management of vaginal agenesis is to provide a vagina of an appropriate length, adequate space without scarring. Careful dissection in the midline, haemostasis and regular use of moulds especially during periods of sexual inactivity to attain optimal result is necessary. Role of vaginal mould is important in graft up take and functional result. AbbeMcIndoe procedure is simple and the results are reproducible. Vaginoplasty by McIndoe technique is successful, safe with satisfactory long term results.

\section{Funding: No funding sources \\ Conflict of interest: None}

Ethical approval: The study was approved by the institutional research review board \& ethics committee and written consent was taken from patient.

\section{REFERENCES}

1. Sultan C, Biason-Lauber A, Philibert P. MayerRokitansky-Kuster-Hauser syndrome: recent clinical and genetic findings. Gynecol Endocrinol. 2009;25(1):8-11.

2. Morcel K, Camborieux L. Programme de Recherches sur les Aplasies Müllériennes, Guerrier D. Mayer-Rokitansky-Küster-Hauser (MRKH) syndrome. Orphanet J Rare Dis. 2007;2:13.

3. Mobus VJ, Kortenhorn K, Kreienberg R, Freidberg V. Long term results after operative correction of vaginal aplasia. Am J Obstet Gynecol. 1996;175:617-24.

4. Azoury RS, Jones HW Jr. Cytogenetic findings in patients with congenital absence of the vagina. Am J Obstet Gynecol. 1966;94:178-80.

5. Sarto GE. Cytogenetics of fifty patients with primary amenorrhea. Am J Obstet Gynecol. 1974;119:14-23.

6. Duncan PA, Shapiro LR, Stangel JJ, Klein RM, Addonizio JC. The MURCS association: Mullerian duct aplasia, renal aplasia, and cervicothoracic somite dysplasia. J Pediatr. 1979;95:399-402. 
7. Leduc B, van Campenhout J, Simard R. Congenital absence of the vagina. Observations on 25 cases. Am J Obstet Gynecol. 1968;100:512-20.

8. Fraser IS, Baird DT, Hobson BM, Michie EA, Hunter W. Cyclical ovarian function in women with congenital absence of the uterus and vagina. J Clin Endocrinol Metab. 1973;36:634-7.

9. Shane JM, Wilson EA, Schiff I, Naftolin F. A preliminary report on gonadotropin responsivity in the Rokitansky-Kuster-Hauser syndrome (congenitally absent uterus). Am J Obstet Gynecol. 1977;127:326-7.

10. Jones KL. Rokitansky sequence. In Smith's recognizable patterns of human malformations (fourth ed). Edited by Saunders WB, Philadelphia. 1988;570-1.

11. Strubbe EH, Willemsen WN, Lemmens JA, Thijn CJ, Rolland R. Mayer-Rokitansky-Kuster-Hauser syndrome: distinction between two forms based on excretory urographic, sonographic, and laparoscopic findings. Am J Roentgenol. 1993;160:331-4.

12. Hensle TW, Chang DT. Vaginal reconstruction. Urol Clin North Am. 1999;26(1):39-47.

13. Hensle TW, Shabsigh A, Shabsigh R, Reiley EA, Meyer-Bahlburg HF. Sexual function following bowel vaginoplasty. J Urol. 2006;175(6):2283-6.

14. Fedele L, Bianchi S, Frontino G, Fontana E, Restelli E, Bruni V. The laparoscopic Vecchietti's modified technique in Rokitansky syndrome: anatomic, functional, and sexual long-term results. Am J Obstet Gynecol. 2008;198(4):377.

Cite this article as: Swain S, Dilip A, Singh P, Satpat hy RN. Vaginal reconstruction with the Abbe-McIndo e technique in Mayer Rokitansky Kuster Hauser syndrome: a case report. Int J Reprod Contracept Obstet Gynecol 2015;4:1602-6. 